\title{
Resultados del clipaje aneurismático temprano en pacientes con hemorragia subaracnoidea de alto grado en el Hospital Regional de Talca
}

Ariel Varela Hernández¹, Félix Orellana Cortéz¹, Claudio Martínez Terreu ${ }^{1}$, Luis Lamus Aponte ${ }^{1}$, Patricio Herrera Astudillo Rodolfo Muñoz Gajardo', Reinaldo Torres Aravena1', Licenciada Cynthia Armijo Olivo'1

1 Servicio de Neurocirugía. Hospital Regional de Talca, Maule, Chile.

Rev. Chil. Neurocirugía 44: 15-24, 2018

\begin{abstract}
Resumen
Introducción: Aproximadamente el $40 \%$ de los pacientes que debutan con Hemorragia Subaracnoidea de causa aneurismática se presentan en pobre condición clínica, con mortalidad que se aproxima al 100\%. Las pautas de atención de estos enfermos son aun controvertidas. Dentro de dichas controversias resalta el tema de la utilidad o no del clipaje aneurismático temprano. En este artículo se analizan los resultados de una serie de pacientes operados. Material y Método: Se estudió una muestra no probabilística de los pacientes operados por el autor principal, con aneurismas intracraneales rotos en grado IV o V (WFNS), en las primeras 72 horas del debut, desde el 1 de enero de 2015 al 31 de diciembre de 2016, en el Hospital Regional de Talca, Maule. Se aplicaron técnicas de estadística descriptiva. Resultados: Se estudiaron 17 pacientes. La mortalidad general fue del $29,4 \%$ y el $41,2 \%$ se registró con buenos resultados de acuerdo a la escala de Rankin modificada. Conclusiones: El clipaje aneurismático es factible de realizarse en estos pacientes con pobre condición clínica, sin embargo, un alto número persiste con discapacidad severa. Se requieren estudios que comprueben la utilidad de estrategias más efectivas para la selección de los pacientes a operar.
\end{abstract}

Palabras clave: Aneurisma intracraneal roto, hemorragia subaracnoidea aneurismática, cirugía, pronostico.

\begin{abstract}
Introduction: Approximately $40 \%$ of patients with Subarachnoid Hemorrhage due to aneurysmal rupture are presented in poor clinical condition, with a mortality approaching $100 \%$. The management of these patients are still controversial. Among these controversies, highlights the issue of the utility or not of early aneurysmal clipping. In this article we analyze the results of a series of patients operated on. Material and Method: We studied a non-probabilistic sample of patients operated by the main author, with ruptured intracranial aneurysms in grade IV or V (WFNS), in the first 72 hours of the debut, from January 1, 2015 to 31 December 2016, at the Regional Hospital of Talca, Maule. Descriptive techniques were applied. Results: We studied 17 patients. Overall mortality was $29.4 \%$ and $41.2 \%$ was recorded with good results according to the modified Rankin scale. Conclusions: Aneurysmal clipping is feasible in these patients with poor clinical condition, however a high number persists with severe disability. Studies that prove the utility of more effective strategies for the selection of patients to be operated are required.
\end{abstract}

Key words: Rupture intracranial aneurysm, aneurysmal subarachnoid hemorrhage, surgery, prognosis.

\section{Introducción}

La hemorragia subaracnoidea aneurismática (HSAa) constituye una enti- dad de gran relevancia en la medicina contemporánea. La misma representa aproximadamente el $10 \%$ de todos los casos detectados con enfermedades cerebrovasculares y se detecta fundamentalmente en personas con edades sociales activas ${ }^{25}$.

A pesar de los tremendos avances in- 
troducidos en la práctica clínica en las últimas décadas; encaminadas a un diagnóstico más oportuno, atención neurointensiva y técnicas de tratamiento de menor invasividad; la historia natural de la enfermedad se mantiene con gran agresividad y elevados índices de morbilidad y mortalidad ${ }^{23,26}$.

Dentro de este conjunto de enfermos, un subgrupo que representa aproximadamente el $40 \%$ de los mismos, ubicado en el extremo más crítico del diapasón de las escalas clínicas de HSAa vigentes (grados IV o V), tienen una situación aún más comprometida y sombría. La utilidad del clipaje aneurismático temprano (en las primeras 72 horas del debut de la hemorragia) en los mismos, ha sido puesto en duda por muchos autores con las fundamentaciones de existencia de lesión neurológica catastrófica y la complejización técnica de la exposición y clipaje aneurismáticos. Además, el desarrollo de las técnicas endovasculares, que evitan la manipulación del encéfalo, motiva que sean preferidas por muchos en estas circunstancias ${ }^{4}$.

De manera contraria, la posición de la neurocirugía contemporánea se inclina cada vez más a conductas agresivas con estos pacientes que garanticen el aislamiento del aneurisma de la circulación sistémica antes de producirse el resangramiento, el lavado del espacio subaracnoideo de sustancias espasminógenas; así como el control de la hipertensión endocraneana mediante la evacuación de hematomas, drenaje de líquido cefalorraquídeo (LCR) o craniectomías descompresivas. No obstante, un número aún muy elevado de los mismos fallecen o se mantienen con secuelas muy invalidantes, elevando ostensiblemente el costo social ${ }^{31}$.

La selección del momento de realización de la cirugía en estas condiciones se mantiene como un tema álgido y no existen estándares aceptados a nivel mundial ${ }^{12}$. En opinión del autor el clipaje aneurismático temprano en pacientes con HSAa de alto grado estaría justificado sobre bases selectivas, por lo que se requieren aún de estudios que demuestren herramientas predictivas efectivas para dirigir de forma más racional estas conductas.

Para fundamentar esta idea se realizó este trabajo que persigue mostrar los resultados de una pequeña serie de pacientes con HSAa operados en grados IV o V en el Hospital Regional de Talca.

\section{Objetivos}

General:

Evaluar los resultados quirúrgicos de los pacientes con hemorragia subaracnoidea aneurismática de alto grado, en los que se realizó clipaje aneurismático temprano.

\section{Específicos:}

1. Precisar la utilidad de la cirugía para lograr la oclusión aneurismática.

2. Reconocer las principales complicaciones que se presentaron en los pacientes.

3. Determinar el estado de los pacientes al finalizar el proceso de atención hospitalaria.

\section{Material y Método}

\section{Diseño y contexto:}

Se realizó un estudio observacional, descriptivo, retrospectivo y transversal con los pacientes atendidos con HSAa en grado IV o $\mathrm{V}$ según la clasificación de la Federación Mundial de Cirujanos Neurológicos (WFNS), a los que se le practicó oclusión microquirúrgica del aneurisma en las primeras 72 del debut del ictus, en el Hospital Regional de Talca, Maule, desde el primero de enero de 2015 al 31 de diciembre de 2016.

\section{Muestra:}

Se creó una muestra no probabilística usando los criterios siguientes:

Criterios de inclusión: Pacientes mayores de 18 años de edad, pacientes detectados con HSAa grado IV o $\mathrm{V}$ según escala de la WFNS (Anexo 1) en el momento de la decisión de la oclusión microquirúrgica del aneurisma, pacientes en los que dicho procedimiento neuroquirúrgico se realizó en las 72 horas subsiguientes al debut del ictus en el Hospital Regional de Talca por el autor, acompañado por un equipo mul- tidisciplinario.

Criterios de exclusión: Pacientes fallecidos antes de arribar al citado centro hospitalario o durante la reanimación en el servicio de urgencias del mismo, pacientes con aneurismas rotos asociados a otras lesiones vasculares estructurales intracraneales (Ejemplo Malformación arterio venosa, etcétera), pacientes con aneurismas de probable causa infecciosa o traumática, pacientes excluidos de la cirugía; lo cual estuvo relacionado con estado moribundo, comorbilidades graves descompensadas o que conferían expectativa de vida inferior a 6 meses, Glasgow postreanimación inferior a 6 puntos o respuesta motora inferior a 4 puntos en dicha escala (por la asunción de lesiones primarias del encéfalo que comprometen la recuperación del paciente), lesiones encefálicas isquémicas relevantes en la tomografía computarizada (TC) de cráneo o no aporte del consentimiento informado por los familiares, así como los pacientes derivados a otros centros hospitalarios para su solución quirúrgica mediante técnicas endovasculares.

\section{Recolección de la información:}

Se revisaron las declaraciones de enfermedad AUGE con el diagnóstico de HSAa en el periodo de tiempo y centro hospitalario antes referidos. Las fichas clínicas de cado uno de ellos, incluyendo los informes imagenológicos y los protocolos operatorios, fueron revisadas por el autor. En aquellos casos en que parte de la evolución posterior a la cirugía se llevó a cabo en otro centro hospitalario o que no presentaron consultas de control en los últimos 3 meses, se contactó al paciente o familiares de forma presencial o telefónica. Con los datos obtenidos se completó un cuestionario confeccionado al efecto.

Proceso de atención hospitalaria: En los pacientes estudiados se siguie-
Anexo 1.

Escala de HSA de Federación Mundial de Cirujanos Neurológicos (WFNS)

\begin{tabular}{|c|c|c|}
\hline Grado & Glasgow & Defecto motor \\
\hline I & 15 & No \\
\hline II & $14-13$ & No \\
\hline III & $14-13$ & $\mathrm{Si}$ \\
\hline IV & $12-7$ & Si/No \\
\hline V & $3-6$ & Si/No \\
\hline
\end{tabular}


ron las recomendaciones de la Guias GES vigentes en Chile $^{34}$ para la atención de los pacientes con HSAa y que en relación al presente estudio se sistematizaron de la siguiente forma:

Período pre oclusión del aneurisma: Incluyó la reanimación cardioventilatoria cuando fue requerida, intubación endotraqueal en pacientes con escala de Glasgow menor a 9 puntos, disritmia ventilatoria o hipoxemia, diagnóstico de la HSA con TC de cráneo simple, urgente. Hospitalización en servicio de paciente crítico, tratamiento farmacológico con fluidoterapia, analgésicos, control de la presión arterial sistólica entre 120 y $159 \mathrm{~mm} \mathrm{Hg}$, nimodipina 60 mg v.o c/4 h, Fenitoína I.V o V.O o Levetiracetam V.O y antiácidos; mantención del tratamiento con estatinas en los casos con indicación previa. Diagnóstico de aneurisma intracraneal roto mediante Angio TC cerebral urgente. Se realizó ventriculostomía al exterior urgente en los casos con hidrocefalia aguda o para el monitoreo continuo de la presión intracraneal (PIC). En los casos con hematoma intraparenquimatosos cerebrales se realizó la evacuación de los mismos durante la cirugía para la oclusión del aneurisma.

Oclusión del aneurisma: Se escogieron abordajes fronto latero pterionales o paramedianos interhemisféricos de acuerdo a la localización del aneurisma roto. En todos los casos se excluyó el aneurisma mediante clipaje con el apoyo del microscopio quirúrgico. También se realizó evacuación de hematomas cuando correspondió y el lavado abundante del espacio subaracnoideo con suero isotónico estéril en todos los casos. La realización de ventriculostomía externa durante el proceder se llevó a cabo en algunos pacientes cuando fue necesario para favorecer la relajación cerebral. El empleo de clipaje arterial transitorio y la apertura de la lámina terminalis se realizó de acuerdo a la decisión del cirujano en cada caso en particular.

Período post oclusión del aneurisma: Se realizó en servicio de atención a pacientes críticos. Se mantuvo en todos los casos al paciente sedado, con ventilación mecánica durante un período de tiempo determinado por la evolución de cada caso. Se aplicó hipertensión euvolémica ligera (presión arterial media de 100 a $110 \mathrm{~mm} \mathrm{Hg}$ ) y Nimodipina $60 \mathrm{mg} \mathrm{c} / 4 \mathrm{~h}$ por vía enteral hasta cumplir 21 días de tratamiento. En los casos con ventriculostomía externa se mantuvo el monitoreo continuo de la PIC, además se realizó el monitoreo con índice biespectral e imagenológico estructural con TC y Angio TC cerebrales como criterio final de la adecuada exclusión del aneurisma de la circulación o detección de vasoespasmo cerebral. En los casos complicados con Defecto Isquémico Tardío (DIT) se aumentó el umbral de hipertensión euvolémica y en las lesiones isquémicas con efecto de masa se procedió con la craniectomía descompresiva. A pesar de la consideración en algunos casos para Angioplastia Transluminar, la misma no pudo realizarse en ninguno de estos casos ya que el estado clínico

\begin{tabular}{|c|c|}
\hline Variables & Codificación \\
\hline Grupos de edades & $\begin{array}{l}18-50 \text { años }=1 \\
51-70 \text { años }=2 \\
\text { mayor a } 70 \text { años }=3\end{array}$ \\
\hline Sexo & $\begin{array}{l}\text { masculino }=1 \\
\text { femenino }=2\end{array}$ \\
\hline Comorbilidad & $\begin{array}{l}\text { sin comorbilidad }=1 \\
\text { comorbilidad sin hipertensión arterial }(\mathrm{HTA}) \\
=2 \\
\text { HTA }=3 \\
\text { HTA asociada a Diabetes Mellitus }(\mathrm{DM})=4 \\
\text { múltiples asociadas a HTA }=5\end{array}$ \\
\hline Síntomas de aviso & $\begin{array}{l}\text { sin síntomas de aviso }=1 \\
\text { síntomas adecuadamente reconocidos }=2 \\
\text { síntomas no reconocidos }=3\end{array}$ \\
\hline $\begin{array}{l}\text { Escala de Glasgow (valor detectado en el } \\
\text { último examen clínico antes de la oclusión } \\
\text { del aneurisma) }\end{array}$ & $\begin{array}{l}12-9 \text { puntos }=1 \\
\text { menor a } 9 \text { puntos }=2\end{array}$ \\
\hline $\begin{array}{l}\text { Escala de la WFNS (valor detectado en el } \\
\text { momento de la decisión de la oclusión del } \\
\text { aneurisma) }\end{array}$ & $\begin{array}{l}I V=1 \\
V=2\end{array}$ \\
\hline $\begin{array}{l}\text { Escala de Fisher modificada (según el } \\
\text { último examen tomográfico antes de la } \\
\text { oclusión del aneurisma) (Anexo 2) }\end{array}$ & $\begin{array}{l}\text { II }=1 \\
\text { III = } 2 \\
\text { IV = } 3 \\
\text { Para el caso de la regresión logística se } \\
\text { usó una codificación dicotómica: } \\
\text { II = } 1 \\
\text { III-IV: } 2\end{array}$ \\
\hline Localización del aneurisma roto & $\begin{array}{l}\text { origen de la comunicante posterior }=1 \\
\text { bifurcación de la carótida interna }=2 \\
\text { bifurcación de la cerebral media }=3 \\
\text { pericalloso }=4 \\
\text { comunicante anterior }=5\end{array}$ \\
\hline Tamaño aneurismático & $\begin{array}{l}0-5 \mathrm{~mm}=1 \\
6-10 \mathrm{~mm}=2 \\
11-24 \mathrm{~mm}=3\end{array}$ \\
\hline Multiplicidad aneurismática & $\begin{array}{l}\text { no }=1 \\
\mathrm{si}=2\end{array}$ \\
\hline
\end{tabular}

impidió el traslado a otros centros distantes para efectuar dicho tratamiento. Se efectuó profilaxis farmacológica de la trombosis venosa profunda con Clexane $40 \mathrm{mg}$ s.c diarios después de 24 horas, con el aval de la TC de cráneo simple. Se aplicaron continuamente técnicas de neurorehabilitación en correspondencia al período evolutivo de los pacientes.

\section{Operacionalización de variables:}

En el cuestionario diseñado se incluyeron las siguientes variables, a las cuales se le aplicó un proceso de codificación, donde el mayor valor se correspondió con la mayor gravedad del evento: 


\begin{tabular}{|c|c|}
\hline $\begin{array}{l}\text { Complicaciones intracraneales previa a la } \\
\text { oclusión }\end{array}$ & $\begin{array}{l}\text { sin complicaciones }=1 \\
\text { convulsión }=2 \\
\text { hidrocefalia }=3 \\
\text { hematoma intraparenquimatoso }(\mathrm{HIP})=4 \\
\text { resangramiento }=5 \\
\text { resangramiento asociado a HIP }=6 \\
\text { Para el caso de la regresión logística se } \\
\text { usó una codificación dicotómica para la } \\
\text { variable Hematoma intraparenquimatoso: } \\
\mathrm{No}=1 \\
\mathrm{Si}=2\end{array}$ \\
\hline $\begin{array}{l}\text { Complicaciones extracraneales previa a la } \\
\text { oclusión }\end{array}$ & $\begin{array}{l}\text { sin complicaciones }=1 \\
\text { broncoaspiración }=2 \\
\text { disritmia ventilatoria }=3 \\
\text { paro cardioventilatorio }=4\end{array}$ \\
\hline Realización de ventriculostomía externa & $\begin{array}{l}\text { no }=1 \\
\text { preoperatoria }=2 \\
\text { transoperatoria }=3 \text { postoperatoria }=4\end{array}$ \\
\hline Momento de la cirugía & $\begin{array}{l}\text { primeras } 24 \text { horas del debut de la } \mathrm{HSA}=1 \\
25-48 \text { horas }=2 \\
49-72 \text { horas }=3\end{array}$ \\
\hline Tipo de clipaje & $\begin{array}{l}\text { simple }=1 \\
\text { múltiple }=2\end{array}$ \\
\hline $\begin{array}{l}\text { Necesidad de reposicionamiento del clip } \\
\text { (durante el propio acto quirúrgico) }\end{array}$ & $\begin{array}{l}\text { no }=1 \\
\text { si }=2\end{array}$ \\
\hline $\begin{array}{l}\text { Empleo de clipaje transitorio del vaso } \\
\text { madre }\end{array}$ & $\begin{array}{l}\text { no }=1 \\
\text { menor o igual a } 5 \text { minutos }=2 \\
6-15 \text { minutos }=3 \\
\text { en dos momentos }=4\end{array}$ \\
\hline Apertura de la lámina terminalis & $\begin{array}{l}\mathrm{si}=1 \\
\mathrm{no}=2\end{array}$ \\
\hline Complicaciones transoperatorias & $\begin{array}{l}\text { sin complicaciones }=1 \\
\text { edema cerebral }=2 \\
\text { rotura aneurismática }=3 \\
\text { Para el caso de la regresión logística se } \\
\text { usó una codificación dicotómica para la } \\
\text { variable Edema cerebral: } \\
\text { No }=1 \\
\mathrm{Si}=2\end{array}$ \\
\hline Complicaciones postoperatorias & $\begin{array}{l}\text { sin complicaciones }=1 \\
\text { neumonía asociada a ventilación }=2 \\
\text { hidrocefalia transitoria }=3 \\
\text { diabetes insípida/natriuresis cerebral }=4 \\
\text { ventriculitis }=5 \\
\text { vasoespasmo sintomático }=6 \text { shock }=7\end{array}$ \\
\hline $\begin{array}{l}\text { Momento en que se registraron los resul- } \\
\text { tados }\end{array}$ & $\begin{array}{l}\text { durante el ingreso }=1 \\
\text { del alta hospitalaria a los } 2 \text { meses }=2 \\
3-12 \text { meses }=3 \\
13-24 \text { meses }=4\end{array}$ \\
\hline Escala de Rankin modificada (Anexo 3) & $\begin{array}{l}\text { I-III (buenos resultados) }=1 \\
\text { IV-VI (malos resultados) }=2\end{array}$ \\
\hline Mortalidad & $\begin{array}{l}\text { vivo }=1 \\
\text { muerte tardía (después del alta hospitalario } \\
\text { o traslado al hospital de base) }=2 \\
\text { temprana (durante la hospitalización) }=3\end{array}$ \\
\hline
\end{tabular}

Procedimientos estadísticos:

Se utilizó el programa S.P.S.S v 18.0, usando técnicas de estadística descriptiva.

\section{Consideraciones éticas:}

La investigación fue avalada por el servicio de Neurocirugía y el Comité de Ética del Hospital Regional de Talca. Las decisiones terapéuticas empleadas se correspondieron con las recomendadas en la literatura universal ${ }^{8,43}$ y las Guías GES vigentes en Chile; en todo caso las mismas se aplicaron de forma consensuada en el servicio de Neurocirugía del Hospital Regional de Talca y con el aval del consentimiento informado del tutor legal del paciente. Los actos quirúrgicos se llevaron a cabo por al menos dos Neurocirujanos y un anestesiólogo con experiencia en procederes neuroanestésicos. La atención pre y postoperatoria se llevó a cabo en Unidades de Pacientes Críticos. Los datos obtenidos a partir de la fichas de los pacientes se mantuvieron en confidensiabilidad en todo momento.

\section{Conflicto de intereses:}

No se declaran conflicto de intereses.

\section{Resultados}

La muestra estuvo integrada por 17 enfermos, que representaron el $43,5 \%$ del universo de 39 pacientes operados de forma temprana por aneurismas intracraneales rotos por el autor, en el período de tiempo estudiado. Se encuestaron pacientes entre 18 y 82 años, la media de la serie fue de 56,1 años. Predominó el sexo femenino con 12 enfermos. La presentación con grado IV de la WFNS se detectó en 10 pacientes y con grado IV de Fisher en 14 de los mismos. Los pacientes estudiados presentaron aneurismas de la circulación anterior, predominaron los originados en el segmento comunicante posterior de la arteria carótida interna con 6 y en la bifurcación de la arteria cerebral media en 5. En 9 pacientes se detectaron aneurismas pequeños $(0-5 \mathrm{~mm})$ y en 7 fueron grandes (11-24 mm). Todos los enfermos fueron operados en las primeras 72 horas del debut de la HSAa, en 3 la oclusión del aneurisma se realizó en las primeras 24 horas. En 12 enfermos se practicó ventriculostomía al exterior, en 7 de los mismos previa a la craneotomía, en 3 durante el abor- 
daje del aneurisma y en 2 en el período postoperatorio. La apertura de la lámina terminalis se efectuó en 4 enfermos. Los pacientes fueron encuestados entre 7 días y 24 meses con una media de 9,9 meses, los valores más bajos se registraron en los enfermos fallecidos. (Tabla 1).

Dentro de las complicaciones preoperatorias (se refiere a la cirugía para oclusión del aneurisma), la hidrocefalia se detectó en 3 pacientes; el hematoma intraparenquimatoso, el resangramiento y la asociación de estos dos últimos, se registró en 2 pacientes en cada caso. Durante el desarrollo de la cirugía se presentó el edema cerebral y la ruptura aneurismática en 3 operados de manera respectiva. En el período postoperatorio se detectó la neumonía asociada a la ventilación mecánica prolongada en 8 enfermos, el vasoespasmo sintomático en 6 y en 1 enfermo la ventriculitis. (Tabla 2).

Se detectaron 5 pacientes $(29,4 \%)$ fallecidos, en 3 de los mismos $(17,6 \%)$ la muerte ocurrió varias semanas después del alta hospitalaria y en 2 $(11,8 \%)$ durante el propio período de hospitalización. Se registraron buenos resultados en la escala de Rankin modificada en 7 (41,2\%). Dentro del grupo de los pacientes con malos resultados se destacan 2 que en la actualidad muestran mejoría progresiva con la rehabilitación. (Figura 1).

\section{Discusión}

Características generales de la serie:

La media de edad de la serie presentada, el predominio del sexo femenino y de aneurismas pequeños, se corresponden con lo publicado en la literatu$\mathrm{ra}^{10,21}$. Llama la atención la indicación quirúrgica en pacientes octogenarios; los resultados alcanzados hoy en día en este tipo de cirugía, conjugados con el envejecimiento poblacional a nivel mundial, han provocado que en relación a la indicación quirúrgica se preste más importancia al estado funcional del paciente y a su expectativa de vida que a su edad cronológica ${ }^{13}$.

Se operaron solo aneurismas de la circulación anterior y dentro de ellos predominaron los del origen de la arteria comunicante posterior y cerebral media, los que junto con los de la arteria comunicante anterior son los más
Tabla 1.

Pacientes según características clínicas. $n=17(43,5 \%)$

\begin{tabular}{|l|l|}
\hline Características & Valores \\
\hline Edad & Máximo: 82 años \\
& Mínimo: 18 años \\
& Media: 56,1 años \\
\hline Sexo & Femenino: $12(70,6 \%)$ \\
& Masculino: $5(29,4 \%)$ \\
\hline Escala de la WFNS & IV: $10(58,8 \%)$ \\
\hline V: $7(41,2 \%)$
\end{tabular}

Tabla 2.

Pacientes según complicaciones

\begin{tabular}{|l|l|}
\hline Complicaciones & Valores \\
\hline Preoperatorias & Hidrocefalia aguda: $3(17,6 \%)$ \\
& Hematoma intraparenquimatoso: 2 \\
& $(11,8 \%)$ \\
& Resangramiento: $2(11,8 \%)$ \\
& Resangramiento con hematoma intra- \\
& parenquimatoso: $2(11,8 \%)$ \\
\hline Transoperatorias & Edema cerebral: $3(17,6 \%)$ \\
& Rotura aneurismática: $3(17,6 \%)$ \\
\hline Postoperatorias & Neumonía asociada a la ventilación: 8 \\
& $(47,1 \%)$ \\
& Vasoespasmo sintomático: $6(35,3 \%)$ \\
& Ventriculitis: 1 (5,9\%) \\
\hline
\end{tabular}

reportados en trabajos similares ${ }^{3,5}$. De forma general en el servicio neuroquirúrgico donde se desarrolló la investigación, los aneurismas de la circu- lación posterior son referidos a otros centros para tratamiento endovascular. La ventriculostomía al exterior, si bien fue usada, no se aplicó en todos los 


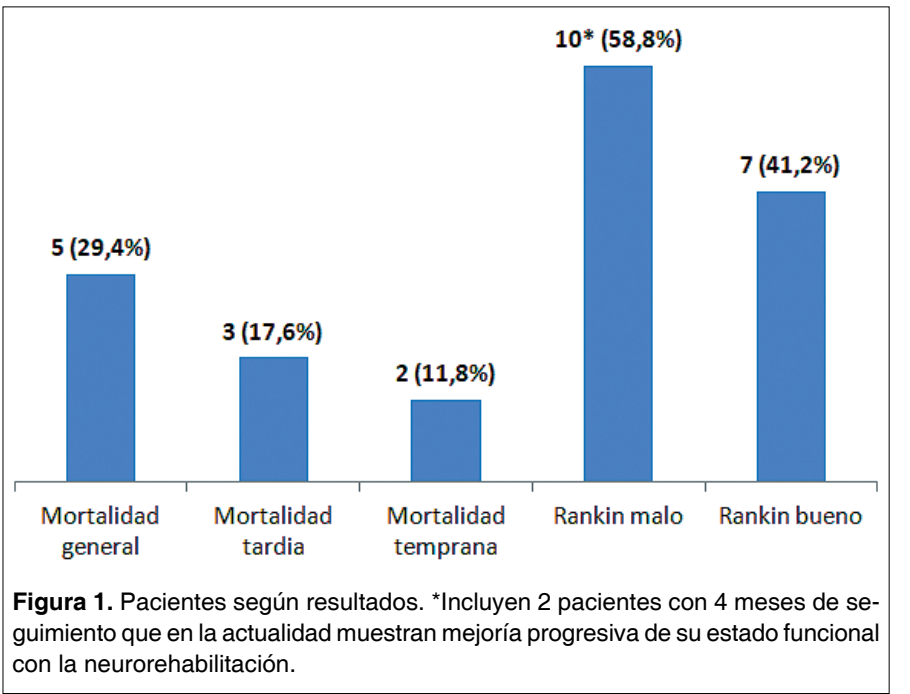

enfermos. En la actualidad esta medida se considera de gran utilidad con el objetivo de aliviar la hipertensión endocraneana inicial y mejorar el estado clínico de estos enfermos. Aunque teóricamente la misma pudiera favorecer el resangramiento por aumento de la presión transmural a nivel del aneurisma, los diferentes estudios publicados la han mostrado como una medida ventajosa en la atención de estos pacientes ${ }^{7}$. Un análisis similar merece la aplicación de apertura de la lámina terminalis que en opinión del autor debe usarse de manera más generalizada en el futuro, procedimiento que algunos estudios han logrado demostrar que disminuye la hidrocefalia shunt dependiente en estos casos, además de contribuir de forma significativa a la reducción transoperatoria del volumen intracranial favoreciendo el acto quirúrgico. Otras técnicas transoperatorias tendrán también que introducirse en dependencia de la disponibilidad en el servicio, como la realización de micro doopler vascular y angiografía con indocianina verde.

En todos los pacientes la cirugía para la oclusión del aneurisma se efectuó dentro de las primeras 72 horas del debut, hecho demostrado como la medida de mayor utilidad en la profilaxis del resangramiento aneurismático; además de ofrecer seguridad para el empleo de tratamientos para la profilaxis o control del defecto neurológico isquémico tardío ${ }^{20}$. Oudshoorn y colaboradores ${ }^{27}$ plantean la utilidad de la cirugía ultratemprana (en las primeras 24 horas) bajo el fundamento de que el momento de mayor riesgo de resangramiento es en las primeras 24 horas, sin embargo aún no llega a demostrarse totalmente las ventajas de esta postura en relación a la cirugía en las primeras 72 horas del debut. El tiempo de evolución medio al momento de la investigación de aproximadamente 10 meses, es favorable para estimar de manera más real los resultados que se muestran.

\section{Análisis de las complicaciones y los resultados:}

En el período preoclusión del aneurisma se encontraron tres complicaciones: la hidrocefalia aguda, el hematoma intraparenquimatoso y el resangramiento. De hecho uno de los aspectos de mayor peso en la definición del mal pronóstico de estos pacientes, es precisamente la mayor incidencia en los mismos de estas graves complicaciones y otras también muy relevantes como la disfunción pulmonar y cardía$\mathrm{ca}^{18,22,41}$

La ruptura aneurismática transoperatoria ocurrió, en los tres pacientes reportados, durante el proceso de disección del aneurisma, en todos fue posible el control de la complicación mediante el empleo de clipaje transitorio del vaso madre, finalización de la disección e instalación del o los clips definitivos. En los casos donde a pesar del drenaje de LCR y el uso de agentes osmolares no se logró una adecuada relajación cerebral antes de iniciar el cierre quirúrgico, se optó por no reponer el flap craneal, ampliar los límites de craneotomía y el empleo de duroplastia expansiva.

En el posoperatorio se presentó un solo caso de ventriculitis, el cual pudo ser resuelto con tratamiento antimicrobiano sistémico e intraventricular. Predominó la presentación de vasosespasmo sintomático, temible complicación también más frecuente en los enfermos con HSAa de alto grado. A pesar del arsenal de técnicas de neuromonitoreo y terapéutico vigente, esta complicación es la de mayor mortalidad y morbilidad en los enfermos con aneurismas ya excluidos y se mantiene como un reto por saldar aun en la atención neurointensiva de los enfermos con $\mathrm{HSAa}^{1,2,11,17,35}$.

Tal y como se describió en el material y métodos, en todos los pacientes se efectuó TAC de cráneo y Angio TAC cerebrales en el postoperatorio, en ningún caso se detectó remanentes aneurismáticos ni oclusiones arteriales relacionados con la aplicación del clip. Es bien reconocido que hasta un $40 \%$ de los pacientes con HSAa se presentan con alto grado y que los resultados generales son aun desfavorables en más del $60 \%$ de los mismos cuando se aplica una atención neuroquirúrgica intensiva, en los que no, la mortalidad supera el $90 \%$, debido a resangramiento en la mayoría de los casos $^{6,44,46,47}$. Los resultados de esta serie en cuanto a mortalidad y grado de discapacidad, medidos con la escala de Rankin modificada, son comparables con lo reportado en la actualidad. Zeng y colaboradore ${ }^{45}$ contrastan en su estudio una mortalidad quirúrgica del $46 \%$ con una del $80 \%$ del grupo de pacientes no operados. Pan y colaboradores ${ }^{28}$ reportaron una mortalidad del $22,2 \%$ y buenos resultados en el $44,4 \%$.

A pesar de que las técnicas endovasculares son preferidas por muchos para la oclusión de los aneurismas rotos en los pacientes con HSAa de alto grado, al evitar la manipulación de un cerebro hostil para la adecuada exposición y tratamiento de la lesión ${ }^{19}$, los resultados que presentamos con la cirugía a cielo abierto son comparables con los de Goel y colaboradores ${ }^{14}$ con mortalidad del $29,1 \%$, malos resultados en el $41,7 \%$ y buenos resultados en el $29,2 \%$.

En esta serie se empleó la cirugía a cielo abierto en todos los casos, en ninguno de ellos las complicaciones surgidas en el trasoperatorio impidieron que se cumpliera el objetivo de la intervención. La literatura sobre el tema comenta que con el empleo del drenaje 
de LCR de las cisternas basales y del sistema ventricular, así como la aplicación de los principios de la neuroanestesia, puede lograrse exponer y excluir el aneurisma roto en la gran mayoría de los casos.

La realización de la craneotomía también permite remover hematomas, la sangre y otras sustancias espasminógenas del espacio subaracnoideo, lo cual contribuye al control de la PIC y a evitar el desarrollo de hidrocefalia y vasoespasmo. Por otro lado se logra la exclusión total y permanente del aneurisma roto en casi el $100 \%$ de los casos $^{42}$ lo que contrasta con aproximadamente un $20 \%$ de recanalizaciones que se registran con el tratamiento endovascular, hecho que aumenta el riesgo de resangramiento y la necesidad de reiteración de tratamientos invasivos. No obstante, no se trata de emular entre ambas opciones, lo mejor es tener la disponibilidad de ambas para indicar la más ventajosa, de acuerdo a las características de cada caso y momento en particular ${ }^{16,24}$.

En relación a los resultados se conoce las ventajas de efectuar el tratamiento en centros que atienden elevados volúmenes de pacientes. En este sentido en el Hospital Regional de Talca, cuyo servicio de Neurocirugía brinda cobertura a toda la región del Maule, una de las más extensas de Chile, se operaron 29 pacientes con aneurismas intracraneales rotos en 2015 y 39 en 2016 , hecho que no lo incluye en los centros de gran volumen de cirugía, los cuales operan al menos 100 aneurismas al año; no obstante en países como Estados Unidos de Norteamérica los centros que operan más de 15 aneurismas al año son certificados para la realización de este tipo de cirugía ${ }^{29}$.

En nuestra opinión encontramos muy útil que en los hospitales regionales existan equipos de trabajo que cumplan con los estándares básicos para la atención integral de estos casos, lo cual disminuye ostensiblemente la posibilidad de complicaciones favorecidas por el tiempo de espera y por el propio traslado de los pacientes, más evidente en los que están en pobre estado neurológico, y optimiza los recursos disponibles, quedando la posibilidad de referencia a centros de atención terciaria para los casos que por su complejidad requieren de la intervención de equipos médicos con mayor experiencia y mayor disponibili- dad de técnicas, como por ejemplo las endovasculares.

Factores de mal pronóstico en pacientes con HSAa de alto grado:

Debido al escaso número de pacientes de la serie presentada y a la dificultad metodológica que genera este hecho para la realización de los análisis estadísticos para evaluar las relaciones de causalidad entre variables, los mismos no pudieron llevarse a cabo en este reporte. A continuación se comentan algunos aspectos relevantes en relación a este tópico reportados en la literatura. El resangramiento preoperatorio constituye la complicación aguda de mayor mortalidad en los pacientes con HSAa $y$ el hecho de ser de alto grado es uno de los factores de riesgo más importante para dicha complicación, cuya incidencia se ha aproximado a un $19 \%$ en varias series publicadas ${ }^{33}$. Rivero-Rodríguez y colaboradores ${ }^{36}$ reportaron una mortalidad en estas condiciones del $61,7 \%$. Por tal motivo, la oclusión temprana del aneurisma se acepta en la actualidad como un eslabón relevante en la atención medica de este grupo de enfermos.

En los trabajos de Pereira y colaboradores $^{30}$ y Zhao y colaboradores ${ }^{48,49}$, este último basado en el estudio multicéntrico de pacientes con HSAa de alto grado, identificado por el acrónimo AMPA, que involucró a 366 pacientes atendidos en varios hospitales Chinos entre los años 2010 al 2012, se relacionó la mayor edad del paciente, ubicación en grado $\mathrm{V}$ de la WFNS, el valor más alto en la escala de Fisher, el vasosespasmo sintomático, la detección de aneurisma de grandes dimensiones o con cuello ancho, la localización en el territorio vértebro basilar y la instalación de neumonía postoperatoria con la mortalidad.

\section{Estrategias en el horizonte:}

A pesar de que los resultados con el tratamiento neuroquirúgico agresivo, que se inicia con la oclusión temprana del aneurisma roto para evitar el resangramiento, mejora de manera significativa la historia natural de la enfermedad en este subgrupo de enfermos con HSAa, los mismos son aun ominosos. Según se demostró en esta serie, concordante con otras ya comentadas en este trabajo, la mortalidad se aproxima al tercio de los pacientes operados y la autovalencia, al menos para satisfacer las necesidades personales, se logra en menos de la mitad de los casos. El hecho de que la disminución de la mortalidad se logre a expensas del aumento del número de pacientes con discapacidad severa, llama la atención sobre la necesidad de nuevas estrategias con mejores dividendos éticos y que al mismo tiempo permitan optimizar los recursos disponibles.

Los pacientes con HSAa de alto grado constituyen en realidad un grupo heterogéneo, con predominio en algunos del compromiso solo funcional y en otros de lesiones estructurales del encéfalo, establecidas por alteraciones en el flujo sanguíneo regional de causa multifactorial, tales como: hipertensión endocraneana, pérdida de la autorregulación vascular, vasoespasmo ultratemprano o de la microcirculación, depresión eléctrica propagada y microtrombosis vascular, entre otros ${ }^{40}$.

En este sentido se han logrado avances en la selección de los pacientes que serán tratados mediante cirugía temprana. A pesar de que la evaluación neurológica partiendo de la suspensión transitoria de los fármacos sedantes, no ha demostrado un aumento en el riesgo de complicaciones y permite identificar a aquellos enfermos que mantienen ciertas funciones neurológicas, sobre todo motoras, esta puede subestimar la existencia de lesiones encefálicas relevantes, mediadas fundamentalmente por la isquemia, que ensombrecerán el pronóstico y por lo tanto hacen menos nítida la ventaja del tratamiento agresivo. Por otro lado, aunque está demostrada la evolución desfavorable en los enfermos que muestran lesiones isquémicas en la TC de cráneo, es bien conocida la baja sensibilidad de esta técnica para detectar dichas lesiones en el estadio agudo, hecho que obliga a la introducción de investigaciones más efectivas ${ }^{9}$.

Dentro de las técnicas que podrían ayudar para definir la existencia de lesión encefálica relevante resaltan en la literatura las siguientes: en el monitoreo con Doppler transcraneal la detección de velocidades en la arteria cerebral media mayores a $200 \mathrm{~cm} /$ segundo, una variabilidad entre exámenes mayor a $50 \mathrm{~cm} / \mathrm{segundo}$ e índice de Lindergard superior a 6 , se corresponden con reducción mayor al $70 \%$ de la luz arterial determinada en la Angio TC cerebral y se relaciona con vasoespasmo sintomático, el cual se asocia con malos 
resultados ${ }^{15,32}$.

La exploración con técnicas para determinar el flujo sanguíneo cerebral, tales como tomografía de emisión de positrones (PET), tomografía de emisión de fotón simple (SPECT), perfusión por RM y TC con Xenón estable (Xe/ $\mathrm{CT}$ ) son útiles para demostrar lesiones isquémicas desde la etapa aguda, sin embargo algunas tienen las desventajas del alto costo, baja disponibilidad o largos períodos de adquisición de imágenes. En este sentido se ha mostrado las ventajas del estudio de la perfusión por TC, disponible en la mayoría de los centros y que requiere poco tiempo de realización; su sensibilidad para detectar lesiones isquémicas encefálicas es de casi el $100 \%$ cuando se registran flujos sanguíneos cerebrales regionales menores a $25 \mathrm{ml} / 100 \mathrm{~g} /$ minuto o tiempos circulatorios medios del encéfalo superiores a 6 segundos ${ }^{37,38}$.
La detección de la disminución de la variabilidad alfa en el monitoreo continuo con electroencefalograma, la caída de la presión tisular de oxígeno del encéfalo por debajo de $20 \mathrm{~mm} \mathrm{Hg}$, índice de Lactato/Piruvato superior a 40 o la detección de lesiones cerebrales extensas en la RM con técnica de difusión se han relacionado también con malos resultados ${ }^{39}$

Aún se requieren de estudios con diseños robustos que prueben la utilidad de estas técnicas, sin embargo, se han publicado diferentes series que permiten evidenciar su probable utilidad para una adecuada selección de los enfermos con HSAa con pobre condición clínica, susceptibles a beneficiarse con la oclusión temprana del aneurisma roto, no solo para evitar la muerte sino también para lograr un resultado funcional aceptable para el individuo, sus familiares y la sociedad.

\section{Conclusiones}

La oclusión temprana de los aneurismas intracraneales rotos en pacientes con HSAa de alto grado es factible de lograrse. También es ventajosa para lograr disminuir de forma significativa la mortalidad en este grupo de enfermos. Sin embargo los resultados muestran aun un índice elevado de los mismos con discapacidad severa, hecho que evidencia la necesidad de profundizar en la aplicación de estrategias que permitan una mejor selección de los pacientes en los que es en realidad ventajoso adoptar esta actitud terapéutica.

Recibido: 16 de noviembre de 2017 Aceptado: 20 de diciembre de 2017

\begin{tabular}{|c|c|}
\hline & $\begin{array}{l}2 . \\
\text { de Fisher modificada }\end{array}$ \\
\hline 0 & No hemorragia subaracnoidea ni intraventricular \\
\hline 1 & Hemorragia subaracnoidea fina (menor a $1 \mathrm{~mm}$ ), sin hemorragia intraventricular \\
\hline 2 & Hemorragia subaracnoidea fina (menor a $1 \mathrm{~mm}$ ), con hemorragia intraventricular \\
\hline 3 & Hemorragia subaracnoidea gruesa (mayor o igual a $1 \mathrm{~mm}$ ), sin hemorragia intraventricular \\
\hline 4 & Hemorragia subaracnoidea gruesa (mayor o igual a $1 \mathrm{~mm}$ ), con hemorragia intraventricular \\
\hline
\end{tabular}

\section{Anexo 3.}

Escala de Rankin modificada

\begin{tabular}{|l|l|}
\hline 0 & Sin síntomas ni limitaciones \\
\hline 1 & $\begin{array}{l}\text { No discapacidad significativa: algunos síntomas pero sin limitaciones para las actividades coti- } \\
\text { dianas y laborales }\end{array}$ \\
\hline 2 & $\begin{array}{l}\text { Discapacidad leve: algunas limitaciones para sus actividades laborales y cotidianas, se mantiene } \\
\text { independiente para las actividades básicas de la vida cotidiana }\end{array}$ \\
\hline 3 & $\begin{array}{l}\text { Discapacidad moderada: satisface sin ayuda las actividades individuales. Ejemplo: alimentación, } \\
\text { aseo, etcétera }\end{array}$ \\
\hline 4 & $\begin{array}{l}\text { Discapacidad moderada - severa: necesita asistencia para las actividades básicas, pero de forma } \\
\text { continua }\end{array}$ \\
\hline 5 & Discapacidad severa: totalmente dependiente, requiere asistencia continua \\
\hline 6 & Muerte \\
\hline
\end{tabular}




\section{Referencias}

1. Athar KM, Levine MJ. Treatment Options for Cerebral Vasospasm in Aneurysmal Subarachnoid Hemorrhage. Neurotherapeutics; 9: 3743. 2012. DOI: 10.1007/s13311-011-0098-1.

2. Castanares-Zapatero $\mathrm{D}$, Hantson Ph. Pharmacological treatment of delayed cerebral ischemia and vasospasm in subaracnoid hemorrhage. Annals of Intensive Care: 12-20. 2011.

3. Castro AM. Understanding the Role of Hemodinamics in the Initiation, Progression, Rupture and Treatment Outcome of Cerebral Aneurysm from Medical Image-Based Computational Studies. ISRN Radiology. 2013 (citado el 3/2/2017) (aprox. 17 páginas). Disponible en: https:// www.researchgate.net/publication/258405155. DOI: 10.5402/2013/602707.

4. Chalouhi N, Jabbour P, Dumont SA, Gonzalez F, Rosenwasser R, Tjoumakaris IS. Endovascular Management of Subarachnoid Hemorrhage. En: Spetzler F R, Kalani M Y, Nakaji P, eds. Neurovascular Surgery. New York: Thieme Medical Publishers. 2015. p.493-504.

5. Chowdhury T, Capellani BR, Sandu N, Schaller B, Daya J. Perioperative Variables Contributieng to the Rupture of Intracranial Aneurysm: An Update. The Scientific World Journal. 2013 (citado el 3/2/2017) (aprox. 7 páginas). Disponible en: PubMed: 2013: 396404. DOI: $10.1155 / 2013 / 396404$.

6. Chua HM, Griessenauer JCh, Thomas JA, Ogilvy SCh. Who is Likely to Present in Poor Neurologic Condition After Aneurysmal Subaracnoid Hemorrhage? Risk Factors and Implications for Treatment. World Neurosurgery; 92: 113-119. 2016.

7. Cohen M, Jethwa P, Prestigiacomo JCh. Subarachnoid Hemorrhage: Workup and Diagnosis. En: Harbaugh ER, Shaffrey Ch, Couldwell TW, Berger SM, eds. Neurosurgery Knowledge Update. New York: Thieme Medical Publishers. 2015. p.39-43.

8. Connolly SE, Rabinstein AA, Carhuapoma RJ, Derdeyn PC, Dion J, Hogashida TR, et al. Guidelines for the Management of Aneurysmal Subarachnoid Hemorrhage: A Guideline for Healthcare Professionals From the American Heart Association/American Stroke Association. Stroke; 43: 1711-1737. 2012.

9. de Oliveira M, Goffi A, Marotta RT, Schweizer AT, Abrahamson S, Macdonald LR. The critical care management of poor-grade subarachnoid haemorrhage. Critical Care; 20: 21-30. 2016. DOI: 10.1186/s13054-016-1193-9.

10. Dong WY, Young JJ, Byung Y Ch, Chul HCh. Subarachnoid Hemorrhage with Negative Baseline Digital Subtraction Angiography: Is Repeat Digital Subtraction Angiography Necessary?. J Cerebrovasc Neurosurg; 14(3): 210-215. 2012.

11. Ducruet FA, Gigante RP, Hickman LZ, Zaharia EB, Arias JE, Grobelny TB. Genetic determinants of cerebral vasospasm, delayed cerebral ischemia, and outcome after aneurysmal subarachnoid hemorrhage. Journal of Cerebral Blood Flow and Metabolism; 30: 676-688. 2010. DOI: $10.1038 / j \mathrm{jbfm} .2009 .278$.

12. Eleftherios A, Nazareno MN. Acute management of poor condition subarachnoid hemorrhage patients. Vascular Health and Risk Management; 3(6): 1075-82. 2007.

13. Ellis AJ, Connolly SE. Ruptured Intracranial Aneurysm: Indications for Microsurgery. En: Harbaugh ER, Shaffrey Ch, Couldwell TW, Berger SM, eds. Neurosurgery Knowledge Update. New York: Thieme Medical Publishers. 2015. p.54-58.

14. Goel G, Gupta V, Chinchure S, Gupta A, Kaur G, Jha NA. A decade after International Subarachnoid Aneurysm Trial: Coiling as a first choice treatment in the management of intracranial aneurysms-Technical feasibility and early management outcomes. Asian $\mathrm{J}$ Neurosurg; 9(3): 137-43. 2014.

15. González-González LJ, Hernández-Zayas H, Bretón-Rosario LJ, Elizondo-Barriel L, López-Arbolay O, Salva-Camaño S, et al. Momento quirúrgico apoyado con Doppler transcraneal en pacientes con hemorragia subaracnoidea aneurismática. Rev. Chil. Neurocirugía; 41: 59-70. 2015.

16. Grasso G, Perra G. Surgical management of ruptured small cerebral aneurysm: Outcome and surgical notes. Surg Neurol Int; 6: 185-190. 2015. DOI: $10.4103 / 2152-7806.171257$.

17. Gupta G, Connolly S. Cerebral Vasospasm and Delayed Ischemic Complications Associatted with Subarachnoid Hemorrhage. En: Spetzler F R, Kalani M Y, Nakaji P, eds. Neurovascular Surgery. New York: Thieme Medical Publishers. 2015. p.478-83.

18. Hatefi M, Azhary S, Naebaghace H, Mohamandi RH, Jaafarpour M. The Effect of Fenestration of Lamina Terminalis on the Vasospasm and Shunt-Dependent Hydrocephalus in Patients Following Subarachnoid Haemorrhage. Journal of Clinical and Diagnostic research; 9(7): 15-18. 2015.

19. Hwang SJ, Hyun KM, Lee JH, Choi EJ, Kim HJ, Lee RN, et al. Endovascular coiling versus neurosurgical clipping in patients with unruptured intracranial aneurysm: a systematic review. BMC Neurology; 12: 99-105. 2012.

20. Ibrahim AAM, Ashmawy GAHO, Eassa AYE, Mansour OY. Hyperacute versus Subacute Coiling of Aneurysmal Sabarachnoid Hemorrhage a Short-term Outcome and Single Center Experience. Pilot Study. Front Neurol; 7: 79-85. 2016. DOI: 10.3389/fneur.2016.00079.

21. Kelliny M, Maeder Ph, Binaghi S, Levivier M, Regli L, Meuli R. Cerebral aneurysm exclusion by CT angiography based on subarachnoid hemorrhage pattern: a retrospective study. BMC Neurology; 11: 8-14. 2011.

22. Kobata H, Sugie A, Yoritsure E, Miyota T, Toho T. Intracranial extravasation of contrast médium during diagnostic CT angiography in the initial evaluation of subarachnoid hemorrhage: report of 16 cases and review of the literature. Springer Plus; 2: 413-22. 2013.

23. Koso M, Dizdarevic K, Sose SJ. Everyday Memory in Microsurgically Treated Patients After Subarachnoid Hemorrhage. J Clin Med Res; 7(4): 225-231. 2015.

24. Kósba GM, Czapiga B, Jarmundowicz W. Aneurismal subarachnoid hemorrhage: who remains for surgical treatment in the post-ISAT era?. Arch Med Sci; 11(3): 536-543. 2015. DOI: 10.5114/aoms.2013.37333.

25. Lanzino G, Rabinstein A A. Subarachnoid Hemorrhage. En: Spetzler FR, Kalani MY, Nakaji P, eds. Neurovascular Surgery. New York: Thieme Medical Publishers. 2015. p.468-77.

26. Lehecka M, Frösen J, Korja M, Lehto H, Kivisaari R, Romani R, et al. Intracranial Aneurysms. En: Spetzler FR, Kalani MY, Nakaji P, eds. Neurovascular Surgery. New York: Thieme Medical Publishers. 2015. p.457-67.

27. Oudshoorn CS, Rinkel EJG, Molyneux JA, Kerr SR, Mess DMS, Backes D, et al. Aneurysm Treatment $<24 \mathrm{~h}$ versus $24-72 \mathrm{~h}$ After Subarachnoid Hemorrhage. Neurocrit Care; 21: 4-13. 2014. DOI: 10.1007/s2028-014-9969-8.

28. Pan J, Zhan R, Wen L, Tong Y, Wan S, Zhon Y. Ultra-Early Surgery for Poor-Grade Intracranial Aneurysmal Subarachnoid Hemorrhage. A preliminary Study. Yosei Med J; 50(4): 521-24. 2009.

29. Pandey SA, Gemmete JJ, Wilson JT, Chaudhary N, Thompson GB, Morgenstern BL, Burke J. High Subarachnoid Hemorrhage Patient Volume Associated with Lower Mortality and Better Outcomes. Neurosurg; 77(3): 462-470. 2015. DOI: 10.1227/NEU.0000000000000850.

30. Pereira OMA, Silva PW, de Figueredo GE, Oliveira AH, Teixeira JM. Fisher revised scale for assessment of prognosis in patients with subarachnoid hemorrhage. Arq. Neuro-Psiquiatr; 69(6): 910-13. 2011. DOI: 10.1590/s0004-282x2011000700012.

31. Quian C, Yu X, Chen J, Gu Ch, Wang L, Chen L, et al. Effect of the drainage of cerebrospinal fluid in patients with aneurysmal subarachnoid hemorrhage. Medicine; 95: 41-48. 2016. DOI: 10.1097/MD.0000000000005140.

32. Quintana-Marín L. Vasoespasmo cerebral y déficit isquémico tardío en la hemorragia subaracnoidea aneurismática. Guías para su manejo 
clínico. Rev. Chil. Neurocirugia; 42: 168-173. 2016.

33. Quing SL, Ping Ch, Yuan XL, Zhang YL, Liang HY, Lin SD, et al. Systolic Blood Pressure Variability is a Nobel Risk Factor for Rebleeding in Acute Subarachnoid Hemorrhage. Medicine; 95(11): 1-6. 2016. DOI: 10,1097/MD.0000000000003028.

34. República de Chile, Ministerio de Salud 2007. Guía clínica Hemorragia Subaracnoidea secundaria a rotura de aneurismas cerebrales. V 2.2. 2013.

35. Ringer JA. Cerebral Vasospasm. En: Harbaugh ER, Shaffrey Ch, Couldwell TW, Berger SM, eds. Neurosurgery Knowledge Update. New York: Thieme Medical Publishers. 2015. p.49-53.

36. Rivero-Rodríguez D, Scherle-Matamoros EC, Gutiérrez-Rojas RA, Pérez-Nellar J, Gómez-Viera N. Características clínicas y evolutivas asociados al resangrado en la hemorragia subaracnoidea aneurismática. Rev Cubana Neurol Neurocir; 4(2): 124-9. 2014.

37. Sanelli CP, Ugrec J, Johnson EC, Tan J, Segal ZA, Fink M, et al. Using quantitative CT perfusión for evaluation of delayed cerebral ischemia following aneurysmal suarachnoid hemorrhage. AJNR; 32(11): 2047-2053. 2011. DOI: 10.3174/ajnr.A2693.

38. Sasahara A, Suzuki K, Takahashi Y, Koseki H, Hirota K, Ohbuchi H, et al. Prognostic Assessment of Aneurysmal Subarachnoid Patients with WFNS Grade V by CT Perfusion on Arrival. World Neurosurgery; 92: 1-6. 2016. DOI: 10.1016/J.WNEU. 2016.04.097.

39. Sato K, Shimizu H, Fujimura M, Inoue T, Matsumoto $Y$, Tominaga T. Acute-stage diffusion-weighted magnetic resonance imaging for predicting outcome of por-grade aneurysmal subarachnoid hemorrhage. Journal of Cerebral Blood Flow and Metabolism; 30: $1110-1120$. 2010. DOI: 10.1038/jcbfm.2009.264.

40. Sehba AF, Hou J, Pluta MR, Zhang HJ. The Importance of Early Brain Injury after Subarachnoid Hemorrhage. Prog Neurobiol; 97(1): 14-37. 2012. DOI: 10.1016/j.pneurobio.2012.02.003.

41. Sijercic AS, Brkic H, Audagic H, Surajic J, Hodzic S. Impact of Comorbidity on Early Outcome of Patients with Subarachnoid Hemorrhage Caused by Cerebral aneurysm Rupture. Med Arch; 69 (5): 280-283. 2015. DOI: 10.5455/medarch.2015.69.280-283.

42. Szmuda T, Sloniewski P. Early and long-term outcome of surgically treated giant internal carotid artery aneurysms-comparison with smaller aneurysms. Acta Neurochir; 153: 1611-1619. 2011. DOI: 10. 1007/s00701-011-1021-6.

43. Wright ES. Medical Management of Subarachnoid Hemorrhage. En: Spetzler FR, Kalani MY, Nakaji P, eds. Neurovascular Surgery. New York: Thieme Medical Publishers. 2015. p.484-92.

44. Zang Y, Zhu X, Hou K, Zhao J, Gao X, Sun Y, et al. Clinical outcomes of surgical clipping for intracranial aneurysms in patients with Hunt and Hess grade 4 or 5. Arq.Neuro-Psiquiatr; 74(6): 478-81. 2016. DOI: 10.1590/0004-282x20160064.

45. Zeng S, Yao P, Yu L, Kang D. Keyhole Approach Combined With External Ventricular Drainage for Ruptured, Poor Grade, Anterior Circulation Cerebral Aneurysms. Medicine; 94(51): 1-8. 2015. DOI: 10.1097/MD.0000000000002307.

46. Zhang Q, Ma L, Liu Y, He M, Sung H, Wang X, et al. Timing of operation for poor-grade aneurysmal subaracnoid hemorrhage: study protocol for a randomized controlled trial. BMC Neurology; 13: 108-115. 2013.

47. Zhao B, Tan X, Yang H, Zhenk K, Li Z, Xion Y, et al. A Multicenter prospective study of poor-grade aneurysmal subarachnoid hemorrhage (AMPAS): observational registry study. BMC Neurology; 14: 86-92. 2014.

48. Zhao B, Yang H, Zheng K, Li Z, Xion Y, Tan X, et al. Predictors of good functional outcomes and mortality in patients with severe rebleeding after aneurysmal subarachnoid hemorrhage. Clinical Neurology and Neurosurgery; 144: 28-32. 2016. DOI: 10.1016/j.clineuro.2016.02.042.

49. Zhao B, Yang H, Zheng K, Li Z, Xion Y, Tan X, et al. Preoperative and postoperative predictors of long-term outcome after endovascular treatment of poor-grade aneurysmal subarachnoid hemorrhage. J Neurosurg; 1: 1-8. 2016

Correspondencia:

Dr. Ariel Varela Hernández

Servicio de Neurocirugía Hospital Regional de Talca

varelahernandezariel@gmail.com 